\title{
Régulation médicale : la HAS édicte des recommandations sur la prise en charge d'un appel
}

\author{
Medical regulation: the French National Authority for Health (HAS) \\ lays down recommendations on the management of calls
}

\author{
M. Giroud · D. Pateron \\ (C) SFMU et Springer-Verlag France 2011
}

Un patient confronté à une urgence médicale ne sait pas en évaluer la gravité. Un médecin formé à cela et agissant dans un centre d'appels dédié aux urgences est le mieux à même d'assurer le dialogue téléphonique avec ce patient, de mâ̂triser les règles d'emploi des ressources, de déterminer et de mettre en œuvre, au cas par cas, la solution la mieux adaptée (le « juste soin »). C'est le principe de la régulation médicale développé par les Samu [1]. La Haute Autorité de santé (HAS), à la demande du ministère de la Santé, vient d'édicter des « recommandations de bonne pratique sur les modalités de prise en charge d'un appel de demande de soins non programmés dans le cadre de la régulation médicale » [2]. Ces recommandations marquent, plus clairement que jamais, que l'acte de régulation médicale est un acte médical et qu'il doit faire l'objet d'un enseignement.

\section{Pratique récente}

Il est intéressant de noter que les premiers pas de la régulation médicale en France répondaient, dans les années 1970, à un objectif bien ciblé : il s'agissait pour les Samu de cadrer l'emploi des ambulances de réanimation des services mobiles d'urgence et de réanimation (Smur) que l'on mettait alors en place (d'où le terme de « régulation »). Une fois cet objectif atteint par la régulation médicale, il est vite apparu inopérant de ne gérer qu'une seule ressource, la plus lourde ; cela aboutissait à laisser un trop grand nombre

\footnotetext{
M. Giroud ( $\square)$

Centre hospitalier René-Dubos,

F-95303 Pontoise, France

e-mail : marc.giroud@ch-pontoise.fr

D. Pateron

Hôpitaux universitaires Est-Parisien, Assistance publique-Hôpitaux de Paris, université Pierre-et-Marie-Curie, 184, rue du Faubourg Saint-Antoine, F-75571 Paris cedex 12, France
}

de demandes sans réponse. Le concept du Samu a donc été élargi à la gestion de cas médicaux urgents de tous les niveaux de gravité, avec, en corollaire, la possibilité de mettre en œuvre l'ensemble des ressources mobilisables en urgence. La mise en service du numéro téléphonique 15 pour les appels du grand public au Samu a consacré cette polyvalence. Diverses crises, dont la pandémie grippale de 2009, ont élargi aux situations exceptionnelles le champ de la régulation médicale du Samu. Enfin, la télémédecine, dont la régulation médicale est la composante la plus active, développe ses quatre autres composantes (téléconsultation, téléexpertise, télésurveillance et téléassistance médicales) au sein des centres de régulation médicale ou en réseau avec ces centres.

\section{Un acte dont la nature médicale est à présent reconnue}

Cette extension du concept s'accompagne d'une mutation : le centre de régulation médicale, outil initialement conçu pour limiter au strict nécessaire l'emploi des ambulances de réanimation, est, au fil du temps, devenu un centre d'appels d'urgence dont la finalité n'est plus la protection (régulation) d'une ressource rare, mais la réponse aux besoins du patient par le « juste soin »; l'optimisation de l'emploi des ressources n'étant plus dorénavant qu'un effet secondaire positif, et non plus la finalité même de l'acte de régulation médicale. Cette évolution sur la finalité est capitale ; elle fait accéder la régulation médicale au rang de pratique médicale. Toutefois, cette évolution n'a pas été immédiatement comprise par tous. L'ordre des médecins a longtemps refusé de reconnaître le caractère médical de la régulation médicale, s'opposant, pour ce motif, à l'autorisation d'accès au dossier médical personnel (DMP) pour le médecin régulateur. Cette opposition n'a pas empêché Samu-Urgences de France et la Société française de médecine d'urgence de promouvoir la régulation médicale et son enseignement, de la 
faire reconnaître dans les décrets « urgences » de 2006 comme l'une des trois composantes de la médecine d'urgence, puis de faire modifier la loi pour permettre au médecin régulateur d'accéder au DMP [3-5]. L'approche judiciaire n'a pas été pour rien dans cette reconnaissance du caractère médical de la régulation médicale : le juge fait, en effet, constamment référence à la façon dont les besoins du patient ont été pris en compte et jamais à la façon dont les ressources collectives ont été protégées.

\section{Apport des recommandations de la HAS}

Ces recommandations définissent des règles générales pour le fonctionnement de la régulation médicale. Elles n'entrent pas dans le champ de la pratique médicale couvert par ailleurs [6-9]. Elles ne sont pas fondées sur des preuves et reposent sur un accord professionnel au sein du groupe de travail, après avis du groupe de lecture ${ }^{1}$.

Les points les plus importants de ces recommandations sont les suivants :

- responsabilité du médecin : «Le médecin régulateur est responsable des réponses apportées à tous les appels »;

- organisation spécifique : «La salle de régulation médicale est exclusivement dédiée et adaptée à cette activité »; «La fonction de médecin régulateur est, pendant la période où elle est assurée, exclusive de toute autre fonction $»$

- éventail de réponses : «Un centre de régulation médicale est organisé de façon que l'équipe médicale puisse disposer d'un éventail de réponses, le plus large possible... ";

- sécurité (notamment la nuit) : «Au moins un médecin régulateur est en permanence en fonction, pour traiter les appels reçus par un centre de régulation médicale $»^{2}$;

- possibilité de fermeture, notamment nocturne : «Les centres de régulation médicale garantissent la continuité du service 24 heures/24. Lorsqu'un centre de régulation médicale est fermé, il bascule les appels vers un centre de régulation médicale fonctionnel, dans le cadre d'un protocole d'accord $»$;

- possibilité d'antenne : «Une antenne de régulation médicale est caractérisée par la participation à l'activité de régulation médicale d'un ou plusieurs médecins, sur une période définie et dédiée à cette activité, dans un lieu

\footnotetext{
$\overline{{ }^{1} \text { Bien qu'ayant }}$ participé à l'élaboration de ce document, l'association SOS Médecins a fait part de son désaccord avec certaines recommandations et n'a pas souhaité être associée aux conclusions de ce travail.

${ }^{2}$ Cette recommandation interdit, de fait, que le médecin régulateur soit dans sa chambre de garde et suppose donc, pour la nuit, que deux médecins, au moins, se relaient, avec un temps de repos suffisant.
}

défini et adapté c'est-à-dire comprenant les outils nécessaires à cette activité (téléphone, outil informatique...), à distance d'un centre de régulation médicale, mais en lien structuré avec ce centre. Dans ce cas, le centre de régulation médicale reçoit l'appel et le transmet à un médecin régulateur de l'antenne qui traite l'appel et renvoie au centre de régulation médicale les informations et les décisions relatives à l'appel »;

- réseau et complémentarités : «Une entraide formalisée entre centres de régulation médicale doit permettre la suppléance et/ou le renfort de l'un par l'autre dans certaines situations définies à l'avance, ainsi qu'en cas de crise ou de défaillance technique... La participation des médecins généralistes à la régulation médicale apporte une complémentarité des compétences professionnelles notamment pour la régulation médicale de la permanence des soins ambulatoires. La mise en réseau de plusieurs centres de régulation médicale est de nature à faciliter, si besoin, l'accès ponctuel à des compétences spécialisées (sagefemme, pédiatre, centre antipoison, psychiatre, gériatre, etc.). Par ailleurs, un centre de régulation médicale peut organiser autour de lui un réseau de consultants susceptibles d'apporter un avis spécialisé dans certains cas particuliers $»$;

- suivi : «Le centre de régulation médicale demande au médecin effecteur de le tenir informé de la prise en charge du patient »; « Le dossier n'est clôturé qu'après avoir eu l'assurance que la prise en charge du patient a bien été relayée ou est terminée »; « ... au vu des informations dont il dispose, le médecin régulateur peut à tout moment décider d'une intervention ou de l'engagement de moyens plus importants »; «En cas de seul conseil médical, le suivi est effectué par téléphone auprès du patient ou de son entourage, selon des modalités que le médecin régulateur définit et mentionne dans le dossier de régulation médicale. Dans le cas où un suivi ne lui semble pas nécessaire, le médecin régulateur le précise de façon explicite»;

- traçabilité : «Tout appel concernant un patient reçu au centre de régulation médicale conduit à l'ouverture d'un dossier de régulation médicale. Un résumé du dossier médical informatisé servant de support à la régulation médicale sera inclus dans le DMP lorsque celui-ci sera opérationnel ».

Ces recommandations ont, au total, le double avantage, d'une part, de tirer vers le haut les pratiques de régulation médicale déjà développées, notamment dans les hôpitaux ou les sociétés d'assistance, et, d'autre part, d'interdire, de fait, toute éventuelle velléité de développement de pratiques de la réponse à des appels médicaux non programmés qui ne répondraient pas à l'ensemble de ces recommandations. 


\section{Références}

1. Giroud M (2009) La régulation médicale en médecine d'urgence. Réanimation 18:737-41

2. Haute Autorité de santé (2011) Modalités de prise en charge d'un appel de demande de soins non programmés dans le cadre de la régulation médicale. www.has-sante.fr

3. Décret urgences (2006) in: Code de la santé publique, art R6123-1. www.legifrance.gouv.fr

4. Giroud M (2009) L'accès au dossier médical personnel par le médecin régulateur du Samu. Congrès Urgences 2009 (Paris) 80:807-16
5. Loi DMP (2007) in: Code de la santé publique art L1111-17. www.legifrance.gouv.fr

6. Berthier F (1998) Sémiologie téléphonique des détresses vitales réelles. Rean Urg 7:129

7. Samu de France (2009) Guide d'aide à la régulation au SamuCentre 15. Sfem, Paris, $517 \mathrm{p}$

8. Penverne Y, Jenvrin J, Debierre V, et al (2011) Régulation médicale des situations à risque. Congrès Urgences 2011 (Paris) 89:1015-33

9. Haute Autorité de santé (2009) Prescription médicamenteuse par téléphone (ou téléprescription) dans le cadre de la régulation médicale. www.has-sante.fr 